\title{
COMMENTARY
}

\section{Blood glucose control in the intensive care unit: discrepancy between belief and practice}

\author{
Dirk Vlasselaers* \\ See related research by Preissig and Rigby, http://ccforum.com/content/14/1/R11
}

\begin{abstract}
A survey among pediatric intensive care physicians showed that a great disparity exists between physicians' beliefs regarding hyperglycemia in critically ill patients and their daily practices to screen and treat hyperglycemia. One of the most prominent reasons for hesitating to implement tight glycemic control is the fear of evoking iatrogenic hypoglycemia. Results from ongoing and future studies focusing on both short- and long-term effects of tight glycemic control in broad populations of critically ill children can provide further strong evidence for implementing tight glycemic control. Improving the accuracy of bedside blood glucose measurements and developing reliable computer algorithms to steer insulin infusions can help to overcome the fear of evoking iatrogenic hypoglycemia.
\end{abstract}

In a recent issue of Critical Care, Preissig and Rigby [1] surveyed the attitudes and practice habits among pediatric intensivists in the US regarding hyperglycemia and tight glycemic control (TGC) in critically ill children. The authors report a considerable disparity between the convictions of the attending physicians and their actual daily practices for blood glucose control in the intensive care unit (ICU). Ninety-seven percent of the participants believed that subsets of critically ill adult patients should be treated for hyperglycemia, and $67 \%$ were convinced that subsets of critically ill children would benefit from glycemic control. However, only a minority of the centers have a standard approach for screening and treating hyperglycemia (7\%) and $80 \%$ lack a standard approach to screen for and treat elevated blood glucose levels. This study provides, therefore, good examples of the

*Correspondence: dirk.vlasselaers@uzleuven.be

Department of Intensive Care Medicine, University Hospitals Leuven, Herestraat 49, B-3000 Leuven, Belgium discrepancy between conviction and practice, of the sceptic implementation of available evidence from clinical studies, and of poor adoption of official recommendations in daily practice.

Hyperglycemia and glucose variability occur very frequently during major surgery and critical illness. These metabolic responses are strongly associated with poor outcome in many different medical conditions in adults, children, and neonates. Prospective randomized trials comparing conventional blood glucose management with age-adjusted TGC in adult surgical [2] and medical [3] ICU patients and in pediatric critically ill patients [4] demonstrated a beneficial effect on morbidity and mortality favoring TGC. Although the debate regarding the pursued blood glucose target ranges is ongoing and other studies [5] (albeit with a different study protocol) could not confirm the results of the previous 'Leuven' trials, a majority of the medical community is convinced that blood glucose really matters, that glycemic management and strategy should be performed in critically ill patients, and that excessive hyperglycemia should be avoided.

However, routine and successful implementation of TGC with intensive insulin therapy remains a difficult hurdle to clear in many ICUs. Among the most prominent reasons for this poor implementation are the fear of evoking iatrogenic hypoglycemia and the general belief that hypoglycemia, albeit for a brief period, is more dangerous and harmful than sustained hyperglycemia. This is elegantly demonstrated in the study by Preissig and Rigby [1].

Hypoglycemia can be the result of the lack of accuracy of the used blood glucose measurement devices, the absence or inadequacy of guidelines and protocols to steer the insulin therapy to achieve TGC, or both. Implementing TGC requires frequent, rapidly available, and accurate blood glucose measurements. However, the high level of accuracy of blood glucose measurements obtained in remote central laboratory facilities or with automated blood gas analyzers cannot be reproduced by many available bedside blood glucose devices in the setting of critically ill patients with a disturbed 'milieu 
interne' (for example, acidosis, hypoxia, and hemodilution) [6,7]. The current unavailability of accurate bedside blood glucose measurements in many ICU departments precludes safe, efficient, and widespread implementation of TGC. Current technology research should focus on the development of accurate and easyto-use continuous blood glucose measurement equipment for critically ill patients.

TGC with intensive insulin therapy increases the workload and responsibility for bedside nurses. Frequent blood testing, interpretation of the blood glucose results, and finally adapting the insulin infusion are very demanding for ICU staff. In addition, guidelines and protocols to steer the insulin infusion are mostly rough guides and experience and intuition are therefore mandatory for successful implementation of TGC. In larger ICUs with a broad medical and nursing staff, it can be a real challenge to convince, motivate, and train the personnel to implement TGC, as demonstrated by Preissig and Rigby [1]. To overcome this 'human' factor and to avoid the use of inefficient and impractical guidelines, computerized algorithms taking into account the recent evolution of blood glucose values, the insulin dose, the caloric intake, and perhaps some physiologic and pharmacologic variables can be a substantial aid [8]. Incorporating an accurate continuous blood glucose analyzer validated for critically ill patients and an effective, safe, and validated computer algorithm into a closed loop system can help to avoid harmful clinical errors leading to iatrogenic-induced hypoglycemia and to successful implementation of TGC.

Finally, the results of ongoing and future studies regarding TGC in critically ill adults and children can help to close the gap between physicians' convictions, attitudes, and daily practices and hence improve the implementation of TGC. The long-term effects of TGC on neurologic and cognitive development and organ functions in children are currently being investigated by the Leuven clinical research group. Multicenter prospective randomized controlled trials, like the ongoing $\mathrm{CHiP}$ (Control of Hyperglycemia in Pediatric Intensive Care) trial in the UK, will provide further knowledge about this intriguing topic.

In conclusion, as shown by Preissig and Rigby, a majority of ICU physicians are convinced that different subsets of critically ill patients, whether adults or children, could benefit from TGC and that this affects outcome. However, only a minority of the centers use a standard and uniform approach to screen and treat hyperglycemia. This considerable disparity between beliefs and actual practices is explained, at least partially, by the fear of evoking hypoglycemia. Strong efforts should be made to improve the accuracy of bedside blood glucose measurements in ICU patients and to develop reliable and safe algorithms to steer insulin infusions and avoid iatrogenic hypoglycemia.

\section{Abbreviations}

ICU, intensive care unit; TGC, tight glycemic control.

\section{Competing interests}

The author declares that he has no competing interests.

\section{Published: 5 May 2010}

\section{References}

1. Preissig CM, Rigby MR: A disparity between physician attitudes and practice regarding hyperglycemia in pediatric intensive care units in the United States: a survey on actual practice habits. Crit Care 2010, 14:R11.

2. Van den Berghe $G$, Wouters P, Weekers F, Verwaest C, Bruyninckx F, Schetz M, Vlasselaers D, Ferdinande P, Lauwers P, Bouillon R: Intensive insulin therapy in critically ill patients. N Engl J Med 2001, 345:1359-1367.

3. Van den Berghe G, Wilmer A, Hermans G, Meersseman W, Wouters P, Milants I Van Wijngaerden E, Bobbaers H, Bouillon R: Intensive insulin therapy in the medical ICU. N Engl J Med 2006, 354:449-461.

4. Vlasselaers D, Milants I, Desmet L, Wouters PJ, Vanhorebeek I, van den Heuvel I, Mesotten D, Casaer MP, Meyfroidt G, Ingels C, Muller J, Van Cromphaut S, Schetz M, Van den Berghe $G$ : Intensive insulin therapy for patients in paediatric intensive care: a prospective, randomised controlled study. Lancet 2009, 14:547-556.

5. NICE-SUGAR study investigators, Finfer $S$, Chittock DR, Su SY, Blair D, Foster D, Dhingra V, Bellomo R, Cook D, Dodek P, Henderson WR, Hébert PC, Heritier S, Heyland DK, McArthur C, McDonald E, Mitchell I, Myburgh JA, Norton R, Potter J, Robinson BG, Ronco JJ: Intensive versus conventional glucose control in critically ill patients. N Engl J Med 2009, 360:1283-1297.

6. Vlasselaers D, Van Herpe T, Milants I, Eerdekens M, Wouters PJ, De Moor B, Van den Berghe G: Blood glucose measurements in arterial blood of ICU patients submitted to tight glycemic control: agreement between bedside tests. J Diabetes Sci Technol 2008, 2:932-938.

7. Rice M, Pitkin A, Coursin D: Glucose measurement in the operating room: more complicated than it seems. Anesth Analg 2010, 110:1056-1065.

8. Cordingley JJ, Vlasselaers D, Dormand NC, Wouters PJ, Squire SD, Chassin LJ, Wilinska ME, Morgan CJ, Hovorka R, Van den Berghe G: Intensive insulin therapy: enhanced model predictive control algorithm versus standard care. Intensive Care Med 2009, 35:123-128.

doi:10.1186/cc8984

Cite this article as: Vlasselaers D: Blood glucose control in the intensive care unit: discrepancy between belief and practice. Critical Care 2010, 14:145. 